\title{
Memories of past close encounters in extreme trans-Neptunian space: Finding unseen planets using pure random searches
}

\author{
C. de la Fuente Marcos ${ }^{1}$ and R. de la Fuente Marcos ${ }^{2}$ \\ 1 Universidad Complutense de Madrid, Ciudad Universitaria, 28040 Madrid, Spain \\ e-mail: nbplanet@ucm.es \\ 2 AEGORA Research Group, Facultad de Ciencias Matemáticas, Universidad Complutense de Madrid, Ciudad Universitaria, \\ 28040 Madrid, Spain
}

Received 11 January 2021 / Accepted 3 February 2021

\begin{abstract}
Context. The paths followed by the known extreme trans-Neptunian objects (ETNOs) effectively avoid direct gravitational perturbations from the four giant planets, yet their orbital eccentricities are in the range between 0.69-0.97. Solar system dynamics studies show that such high values of the eccentricity can be produced via close encounters or secular perturbations. In both cases, the presence of yet-to-be-discovered trans-Plutonian planets is required. Recent observational evidence cannot exclude the existence, at $600 \mathrm{AU}$ from the Sun, of a planet of five Earth masses.

Aims. If the high eccentricities of the known ETNOs are the result of relatively recent close encounters with putative planets, the mutual nodal distances of sizeable groups of ETNOs with their assumed perturber may still be small enough to be identifiable geometrically. In order to confirm or reject this possibility, we used Monte Carlo random search techniques.

Methods. Two arbitrary orbits may lead to close encounters when their mutual nodal distance is sufficiently small. We generated billions of random planetary orbits with parameters within the relevant ranges and computed the mutual nodal distances with a set of randomly generated orbits with parameters consistent with those of the known ETNOs and their uncertainties. We monitored which planetary orbits had the maximum number of potential close encounters with synthetic ETNOs and we studied the resulting distributions.

Results. We provide narrow ranges for the orbital parameters of putative planets that may have experienced orbit-changing encounters with known ETNOs. Some sections of the available orbital parameter space are strongly disfavored by our analysis.

Conclusions. Our calculations suggest that more than one perturber is required if scattering is the main source of orbital modification for the known ETNOs. Perturbers might not be located farther than $600 \mathrm{AU}$ and they have to follow moderately eccentric and inclined orbits to be capable of experiencing close encounters with multiple known ETNOs.
\end{abstract}

Key words. methods: data analysis - methods: numerical - celestial mechanics - planets and satellites: detection minor planets, asteroids: general - Kuiper belt: general

\section{Introduction}

Extreme trans-Neptunian objects (ETNOs) serve as unique probes into the gravity perturbations shaping the outer solar system beyond the classical trans-Neptunian or Kuiper belt (see e.g., Kaib et al. 2019). The trajectories followed by the known ETNOs effectively avoid direct gravitational perturbations from the four giant planets, yet their orbital eccentricities are in the range 0.69-0.97. Efficient drivers for the eccentricity excitation of small bodies include close encounters with planets (see e.g., Carusi et al. 1990) and the von Zeipel-Lidov-Kozai mechanism (von Zeipel 1910; Kozai 1962; Lidov 1962; Ito \& Ohtsuka 2019). In both cases above and in the case of ETNOs, the presence of yet-to-be-discovered trans-Plutonian planets is required.

Fienga et al. (2020) used the INPOP19a planetary ephemerides that include Jupiter-updated positions by the Juno mission and a reanalysis of Cassini observations to show that there is no clear evidence for the existence of the so-called Planet 9 predicted by Batygin \& Brown (2016) as an explanation for the orbital architecture of the known ETNOs. However, Fienga et al. (2020) concluded that if Planet 9 exists, it cannot be closer than $500 \mathrm{AU}$, if it has a mass of $5 M_{\oplus}$, and no closer than $650 \mathrm{AU}$, if it has a mass of $10 M_{\oplus}$. The latest version of the Planet 9 hypothesis (Batygin et al. 2019) predicts the existence of a planet with a mass in the range 5-10 $M_{\oplus}$, following an orbit with a value of the semi-major axis in the range of 400-800 AU, eccentricity in the range of $0.2-0.5$, and inclination in the interval between $\left(15^{\circ}, 25^{\circ}\right)$. A number of exoplanets have already been observed orbiting at hundreds of AUs from their host stars (see e.g., Bailey et al. 2014; Naud et al. 2014; Nguyen et al. 2021) and theoretical calculations have confirmed plausible pathways for their formation (see e.g., Kenyon \& Bromley 2015, 2016).

If the high eccentricities of known ETNOs are the result of relatively recent close encounters with putative planets, the mutual nodal distances of sizeable groups of ETNOs with their assumed perturber may still be small enough to be identifiable geometrically. Here, we use Monte Carlo random search techniques to identify orbits that may lead to the maximum number of potential close encounters with synthetic ETNOs whose orbital parameters are consistent with those of the real ETNOs and their uncertainties. This Letter is organized as follows. In 
Sect. 2, we review our methodology and present the data used in our analyses. In Sect. 3, we apply our methodology and discuss its results. Our conclusions are summarized in Sect. 4.

\section{Methods and data description}

This work explores a "what if" scenario in which the starting hypothesis states that a sizeable number of known ETNOs have experienced relatively recent close encounters with putative planets; the timescale comes constrained by their orbital periods that are in the range 1867-50116yr, so encounters may have taken place during the last $10^{3}-10^{5} \mathrm{yr}$. In this work, therefore, we are testing the hypothesis statistically. If this hypothesis is plausible, a statistically significant number of compatible planetary perturber orbits should emerge from the analysis of a very large sample of orbits. If the distributions of one or more of the orbital parameters of the candidate are flat, the starting hypothesis must be rejected as this would show that there is no favored orbital solution for the perturber; conversely, if all the distributions produce consistent intervals that are statistically significant, the plausibility of the starting hypothesis can be considered as confirmed. Plausibility concerns the likelihood of acceptance, not the likelihood of being true or better than competing scenarios. The problem under investigation here is equivalent to a nondifferenciable optimization that is well-suited for a uniform Monte Carlo random search (Metropolis \& Ulam 1949). Our methodology brings together geometry and statistics in our attempt to find the confocal ellipse that passes the closest to the maximum number of known orbits of a certain dynamical class; this approach is fundamentally different from those involving $N$-body calculations and statistics (see e.g., de la Fuente Marcos et al. 2016, 2017; de León et al. 2017). Kalinicheva \& Chernetenko (2020) recently applied a geometric approach within the context of the Planet 9 hypothesis.

\subsection{Methodology}

Two arbitrary orbits may experience close encounters when their mutual nodal distance is sufficiently small. Recurrent (or even single) encounters within 1 Hill radius (Hamilton \& Burns 1992) of a massive body may change the orbit of a small body significantly. The mutual nodal distance between the orbits of a small body (an ETNO in our case) and an arbitrary planet can be computed as described in Appendix A. Orbits are defined by the values of semi-major axis, $a$ (that controls size), eccentricity, $e$ (that controls shape), and those of the angular elements - inclination, $i$, longitude of the ascending node, $\Omega$, and argument of perihelion, $\omega$ - that control the orientation in space of the orbit; the perihelion distance or pericenter, $q$, is given by the expression $q=a(1-e)$. The actual position of an object in its orbit is controled by a fourth angle, the mean anomaly, $M$. Our geometric approach leaves this angle out of the analysis and, therefore, it is not capable of predicting the current location of the perturber, if it is, in fact, real.

We generated $2 \times 10^{10}$ random planetary orbits with uniformly distributed relevant parameters: $\Omega_{\mathrm{p}}$ and $\omega_{\mathrm{p}} \in\left(0^{\circ}, 360^{\circ}\right)$, $i_{\mathrm{p}} \in\left(0^{\circ}, 80^{\circ}\right), e_{\mathrm{p}} \in(0,0.9)$, and $q_{\mathrm{p}} \in(x, 1000-x) \mathrm{AU}$, with $x=300,400,500$, and 600 AU so $a_{\mathrm{p}}=q_{\mathrm{p}} /\left(1-e_{\mathrm{p}}\right)$. For each random planetary orbit, we computed the mutual nodal distances between a set of synthetic ETNOs and the planet. Each set of synthetic ETNOs was generated using the mean values and standard deviations of the orbit determinations of the known ETNOs as pointed out in Appendix B.
For each combination of random planetary orbit and synthetic (but compatible with the observations) set of ETNOs, we have counted how many synthetic ETNOs had at least one mutual nodal distance with the planet under $5 \mathrm{AU}$ (for $x=$ $300 \mathrm{AU}$ ), 7.5 AU (for $x=400 \mathrm{AU}$ ), and $10 \mathrm{AU}$ (for $x=500$ and $600 \mathrm{AU})$. We then proceeded to record the random planetary orbit if the count was $\geq 5$, in order to maximize the number of potential close approaches between planet and set of ETNOs. We then studied the resulting distributions. In order to analyze the results, we produced histograms using the Matplotlib library (Hunter 2007) with sets of bins computed using NumPy (van der Walt et al. 2011) by applying the Freedman and Diaconis rule (Freedman \& Diaconis 1981). Instead of using frequency-based histograms, we considered counts to form a probability density so the area under the histogram will sum to one.

The nodal distance separation criteria for selective counting are not arbitrary but motivated by the results in Fienga et al. (2020), a $2 M_{\oplus}$ has a Hill radius of $4.5 \mathrm{AU}$ (if $a_{\mathrm{p}}=400 \mathrm{AU}$ and $e_{\mathrm{p}}=0.1$ ), a $5 M_{\oplus}$ has a Hill radius of $9.2 \mathrm{AU}$ (if $a_{\mathrm{p}}=542 \mathrm{AU}$ and $\left.e_{\mathrm{p}}=0.01\right)$. Therefore, we implicitly assume that the farther away the pericenter of the planet is, the more massive it may be.

\subsection{Data sources}

Here, we work with publicly available data from Jet Propulsion Laboratory's (JPL) Small-Body Database (SBDB) ${ }^{1}$ and HORIZONS on-line solar system data and ephemeris computation service $^{2}$, both provided by the Solar System Dynamics Group (Giorgini 2015). Assuming the definition in Trujillo \& Sheppard (2014), that the ETNOs have $q>30 \mathrm{AU}$ and $a>150 \mathrm{AU}$, the sample of known ETNOs now includes 39 objects with reliable orbits (see Appendix B) whose data have been retrieved from JPL's SBDB and HORIZONS using tools provided by the Python package Astroquery (Ginsburg et al. 2019). In the following section, we use barycentric elements because within the context of the ETNOs, barycentric orbit determinations better account for their changing nature as Jupiter follows its $12 \mathrm{yr}$ orbit around the Sun; Appendix C shows results based on heliocentric orbits that are consistent with those of the barycentric ones.

\section{Results and discussion}

As pointed out above, the motivation behind this study is the belief that a fossil record of planetary encounters might be preserved in the distribution of the orbital elements of ETNOs. With this hypothesis in mind, we monitored which planetary orbits had the maximum number of potential close encounters with synthetic ETNOs and analyzed the resulting distributions that are shown in Fig. 1. The first (leftmost) column of panels shows the results based on the assumption that $q_{\mathrm{p}}>300 \mathrm{AU}$, including 20304 orbits with a number of potential close approaches in the range between 5-7; the next one, shows results for $q_{\mathrm{p}}>400 \mathrm{AU}$ also with a range of potential close approaches of 5-7 for 5671 orbits; the following column of panels displays results for $q_{\mathrm{p}}>$ $500 \mathrm{AU}$ with a range of 5-6 for 2635 orbits; the right column shows panels with results for $q_{\mathrm{p}}>600 \mathrm{AU}$ and the number of potential close approaches between planet and set of ETNOs is just 5 for 56 orbits. From these values and the plots, it is increasingly difficult to find consistent perturbers as we move farther away from the Sun.

\footnotetext{
1 https://ssd.jpl.nasa.gov/sbdb.cgi

2 https://ssd.jpl.nasa.gov/?horizons
} 

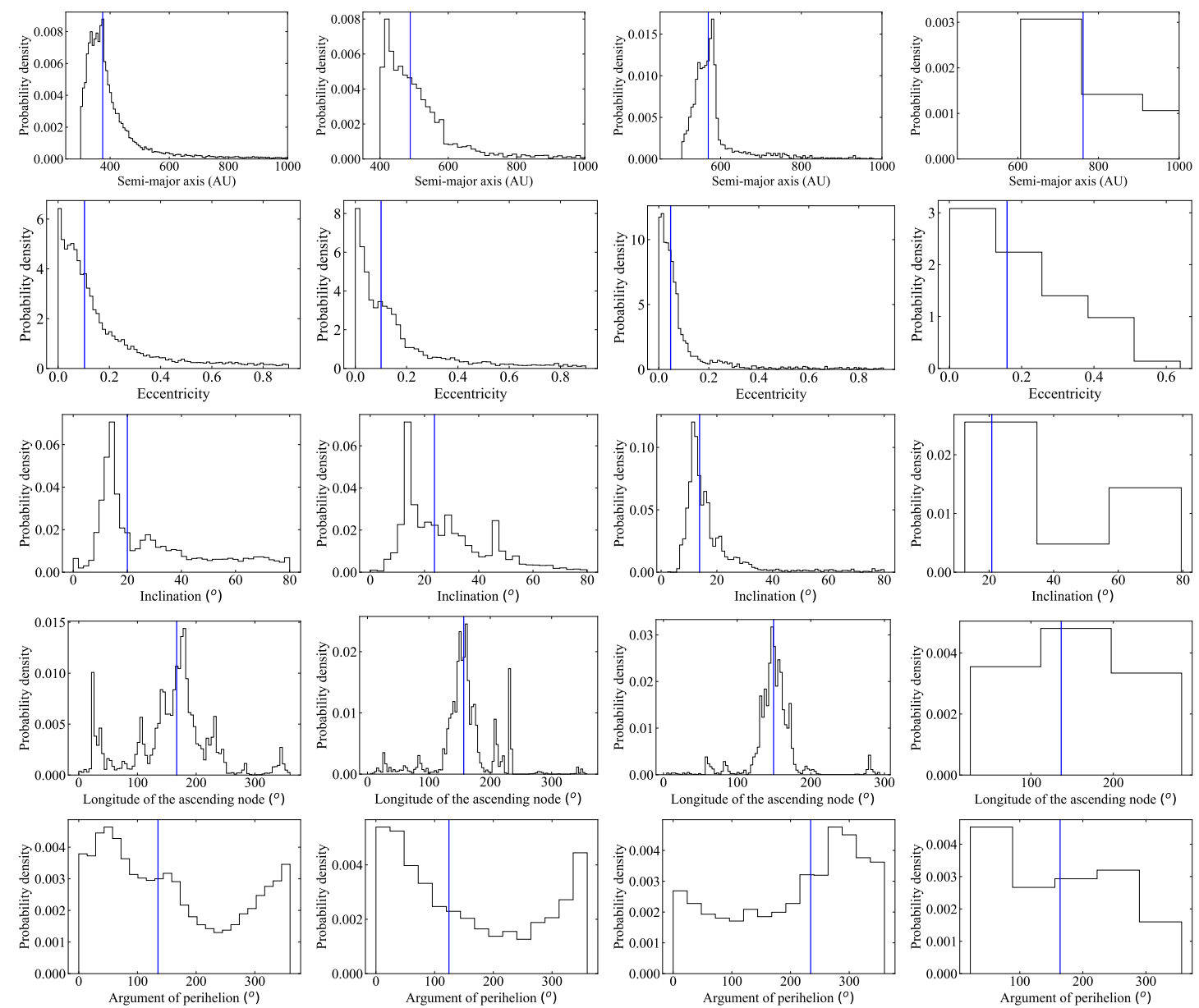

Fig. 1. Barycentric orbital elements of putative perturber planets. Distributions of barycentric orbital elements of planetary orbits that may result in close encounters (under $5 \mathrm{AU}$ for $q_{\mathrm{p}}>300 \mathrm{AU}$, under $7.5 \mathrm{AU}$ for $q_{\mathrm{p}}>400 \mathrm{AU}$, and under $10 \mathrm{AU}$ for $q_{\mathrm{p}}>500 \mathrm{AU}$ and $q_{\mathrm{p}}>600 \mathrm{AU}$ ) with five or more present-day extreme trans-Neptunian objects (ETNOs). Each column of panels shows the cumulative results of $2 \times 10^{10}$ experiments. From left to right: results of imposing $q_{\mathrm{p}}>300 \mathrm{AU}$ (left column, 20304 orbits), $q_{\mathrm{p}}>400 \mathrm{AU}$ (5671 orbits), $q_{\mathrm{p}}>500 \mathrm{AU}$ ( 2635 orbits), and $q_{\mathrm{p}}>600 \mathrm{AU}$ (right column, 56 orbits). Median values are shown as vertical blue lines. Based on the available data on known ETNOs, the presence of massive perturbers well beyond $600 \mathrm{AU}$ is strongly excluded within the context of the hypotheses centered in this study.

Table 1. Summary of central values and dispersions of optimal barycentric orbits.

\begin{tabular}{lcccc}
\hline \hline Orbital parameter & $q_{\mathrm{p}}>300 \mathrm{AU}$ & $q_{\mathrm{p}}>400 \mathrm{AU}$ & $q_{\mathrm{p}}>500 \mathrm{AU}$ & $q_{\mathrm{p}}>600 \mathrm{AU}$ \\
\hline Semi-major axis, $a_{\mathrm{p}}(\mathrm{AU})$ & $375_{-44}^{+111}(376)$ & $489_{-64}^{+130}(420)$ & $569_{-31}^{+55}(581)$ & $762_{-120}^{+279}(680)$ \\
Eccentricity, $e_{\mathrm{p}}$ & $0.10_{-0.07}^{+0.21}(0.01)$ & $0.10_{-0.08}^{+0.19}(0.01)$ & $0.05_{-0.03}^{+0.09}(0.02)$ & $0.16_{-0.13}^{+0.19}(0.07)$ \\
Inclination, $i_{\mathrm{p}}\left(^{\circ}\right)$ & $20_{-8}^{+34}(14)$ & $24_{-10}^{+22}(14)$ & $144_{-3}^{+9}(12)$ & $21_{-6}^{+52}(24)$ \\
Longitude of the ascending node, $\Omega_{\mathrm{p}}\left(^{\circ}\right)$ & $167_{-70}^{+44}(180)$ & $157_{-23}^{+48}(161)$ & $149_{-17}^{+18}(148)$ & $137_{-55}^{+141}(156)$ \\
Argument of perihelion, $\omega_{\mathrm{p}}\left({ }^{\circ}\right)$ & $135_{-94}^{+170}(51)$ & $124_{-94}^{+193}(12)$ & $234_{-165}^{+81}(275)$ & $164_{-106}^{+109}(56)$ \\
\hline
\end{tabular}

Notes. Median values and 16th and 84th percentiles (absolute maximum in parentheses) from the Monte Carlo random searches whose distributions are shown in Fig. 1.

Table 1 shows a summary of the central values and dispersions of the orbital parameters of the sample of orbits that may experience close encounters with multiple known ETNOs. We consider that results for $q_{\mathrm{p}}>300 \mathrm{AU}$ and $q_{\mathrm{p}}>400 \mathrm{AU}$ are statistically consistent and are compatible with $a_{\mathrm{p}} \in(331,489) \mathrm{AU}$, $e_{\mathrm{p}}<0.1, i_{\mathrm{p}} \in\left(10^{\circ}, 20^{\circ}\right), \Omega_{\mathrm{p}} \sim 180^{\circ}$, and $\omega_{\mathrm{p}} \in\left(-60^{\circ}, 60^{\circ}\right)$. The value of $a_{\mathrm{p}}$ is probably $\sim 400 \mathrm{AU}$ but the uncertainty is significant, the value of the eccentricity is well constrained and it has to be low, the inclination is perhaps the best constrained value and it is $\sim 14^{\circ}$, the value of $\Omega_{\mathrm{p}}$ is very likely $\sim 180^{\circ}$, but the value of $\omega_{\mathrm{p}}$ is poorly constrained, perhaps $\sim 50^{\circ}$. If scattering is the main source of orbital modification for the group of ETNOs that mainly move at $(300,400) \mathrm{AU}$ from the Sun, the orbit of the putative perturber is relatively well-constrained and according to Fienga et al. (2020), it must have a mass $<5 M_{\oplus}$.

Table 1 shows that our approach is far less conclusive in the cases of $q_{\mathrm{p}}>500 \mathrm{AU}$ and $q_{\mathrm{p}}>600 \mathrm{AU}$ as these values produce distributions of the orbital parameters that may not be statistically compatible. In any case, we must emphasize that $>50 \%$ of the known ETNOs cannot interact directly 
with a perturber with $q_{\mathrm{p}}>500 \mathrm{AU}$ because their aphelion distances, $Q=a(1+e)$, are below $500 \mathrm{AU}$ : 9 ETNOs have $Q<300 \mathrm{AU}, 18$ have $Q<400 \mathrm{AU}$, and 23 have $Q<500 \mathrm{AU}$. Therefore, the distribution in $Q$ and our calculations suggest that more than one perturber is required if scattering is the main source of orbital modification for the known ETNOs. Perturbers might not be located farther than $600 \mathrm{AU}$ and they have to follow moderately eccentric and inclined orbits to be capable of experiencing present-day close encounters with multiple known ETNOs.

At this point, it can be argued that the results in Fig. 1 may be affected by a statistical artifact. In order to test the statistical significance of our results, we repeated the Monte Carlo random search on an input sample of scrambled data as explained in Appendix D. By randomly reassigning the values of the orbital elements of the ETNOs, we preserve the original distributions of the parameters, but destroy any possible correlations that may have been induced by close encounters with massive perturbers (or the effects of hypothetical mean-motion or secular resonances). The results of this significance test are shown in Fig. D.1: the distribution of $i_{\mathrm{p}}$ becomes flat and almost the same happens to the distribution of $\Omega_{p}$. In other words, for the scrambled data, it is not possible to find a statistically significant orbital solution that is compatible with the starting hypothesis and we conclude that the results in Fig. 1 are unlikely to be due to statistical artifacts.

\section{Summary and conclusions}

When considering the well-studied case of Neptune and the regular trans-Neptunian objects, we observe that these objects are not part of a single population, but they are organized into several dynamical classes. Some objects never experience close encounters with Neptune due to the existence of protection mechanisms such as mean-motion or secular resonances, as in the case of Neptune's Trojans or Pluto (see e.g., Milani et al. 1989; Wan et al. 2001), others undergo close encounters that may send them towards the inner solar system (centaurs) or outwards to become scattered objects (see the recent review by Saillenfest 2020). If trans-Plutonian planets exist, their perturbations may shape the extreme trans-Neptunian space in a similar fashion and the current sample of ETNOs may include the signatures of their presence (see e.g., Saillenfest et al. 2017).

Our results show that it is possible to find suitable orbits for which the mutual nodal distances of sizeable $(\geq 5$, comprising at least $13 \%$ of the sample) groups of ETNOs with their assumed perturber could be small enough for a close encounter to occur, at least in theory (assuming that no protection mechanisms, such as mean-motion or secular resonances, are in place to avoid the flyby). This was our original aim. In addition, the results presented in Sect. 3 clearly indicate that the most probable planetary orbit consistent with the starting hypothesis is the one obtained for the experiment with $q_{\mathrm{p}}>300$ AU. The number of consistent orbits in this case is 3.6 times higher than the one found for $q_{\mathrm{p}}>400 \mathrm{AU}$. Our results seem to be incompatible with those attributed to a statistical artifact (see Appendix D).

Prior to the announcement of the Planet 9 hypothesis (Batygin \& Brown 2016), Trujillo \& Sheppard (2014) had already argued for the existence of a planet at $250 \mathrm{AU}$ within the context of the ETNOs - driving von Zeipel-Lidov-Kozai librations on 2012 VP $_{113}$ - and de la Fuente Marcos \& de la Fuente Marcos (2014) suggested that the limited data available at the time were more compatible with the presence of two massive perturbers, one of them close to $300 \mathrm{AU}$. These massive perturbers were initially proposed based on data corresponding to a sample of 13 objects, whereas the current sample has tripled this number. If we repeat the experiment discussed in Sect. 3 for $q_{\mathrm{p}}>200 \mathrm{AU}$ (see Appendix E), we find that the number of consistent orbits, although larger than the one generated in the experiment for $q_{\mathrm{p}}>400 \mathrm{AU}$, is still lower than that of the most statistically significant case, namely, 8234 versus 20304 for $q_{\mathrm{p}}>300 \mathrm{AU}$. The most likely orbit is still similar, in terms of shape and orientation in space, to the most probable one in the $q_{\mathrm{p}}>300$ AU case.

Our results are consistent with the conclusions of the study presented in de la Fuente Marcos \& de la Fuente Marcos (2017). Although our approach has not been able to single out a statistically significant, unique planetary orbit that may be responsible for the orbital architecture observed in extreme trans-Neptunian space, we provide narrow ranges for the orbital parameters of putative planets that may have experienced orbitchanging encounters with known ETNOs. Some sections of the available orbital parameter space are strongly excluded by the findings of our analysis.

Acknowledgements. We thank the anonymous referee for a constructive and timely report, S. J. Aarseth, J. de León, J. Licandro, A. Cabrera-Lavers, J.-M. Petit, M. T. Bannister, D. P. Whitmire, G. Carraro, E. Costa, D. Fabrycky, A. V. Tutukov, S. Mashchenko, S. Deen and J. Higley for comments on ETNOs and A. I. Gómez de Castro for providing access to computing facilities. This work was partially supported by the Spanish 'Ministerio de Economía y Competitividad' (MINECO) under grant ESP2017-87813-R. In preparation of this Letter, we made use of the NASA Astrophysics Data System and the MPC data server.

\section{References}

Bailey, V., Meshkat, T., Reiter, M., et al. 2014, ApJ, 780, L4

Batygin, K., \& Brown, M. E. 2016, AJ, 151, 22

Batygin, K., Adams, F. C., Brown, M. E., et al. 2019, Phys. Rep., 805, 1

Carusi, A., Valsecchi, G. B., \& Greenberg, R. 1990, Celest. Mech. Dyn. Astron., 49, 111

de la Fuente Marcos, C., \& de la Fuente Marcos, R. 2014, MNRAS, 443, L59 de la Fuente Marcos, C., \& de la Fuente Marcos, R. 2017, MNRAS, 471, L61 de la Fuente Marcos, C., de la Fuente Marcos, R., \& Aarseth, S. J. 2016, MNRAS, 460, L123

de la Fuente Marcos, C., de la Fuente Marcos, R., \& Aarseth, S. J. 2017, Ap\&SS, 362,198

de León, J., de la Fuente Marcos, C., \& de la Fuente Marcos, R. 2017, MNRAS, 467, L66

Fienga, A., Di Ruscio, A., Bernus, L., et al. 2020, A\&A, 640, A6

Freedman, D., \& Diaconis, P. 1981, Prob. Theor. Relat. Fields, 57, 453

Ginsburg, A., Sipőcz, B. M., Brasseur, C. E., et al. 2019, AJ, 157, 98

Giorgini, J. D. 2015, IAU Gen. Assem., 22, 2256293

Hamilton, D. P., \& Burns, J. A. 1992, Icarus, 96, 43

Hunter, J. D. 2007, Comput. Sci. Eng., 9, 90

Ito, T., \& Ohtsuka, K. 2019, Monogr. Environ. Earth Planets, 7, 1

Kaib, N. A., Pike, R., Lawler, S., et al. 2019, AJ, 158, 43

Kalinicheva, O. V., \& Chernetenko, Y. A. 2020, Astrophys. Bull., 75, 459

Kenyon, S. J., \& Bromley, B. C. 2015, ApJ, 806, 42

Kenyon, S. J., \& Bromley, B. C. 2016, ApJ, 825, 33

Kozai, Y. 1962, AJ, 67, 591

Lidov, M. L. 1962, Planet. Space Sci., 9, 719

Metropolis, N., \& Ulam, S. 1949, J. Am. Stat. Assoc., 44, 335

Milani, A., Nobili, A. M., \& Carpino, M. 1989, Icarus, 82, 200

Naud, M.-E., Artigau, É., Malo, L., et al. 2014, ApJ, 787, 5

Nguyen, M. M., De Rosa, R. J., \& Kalas, P. 2021, AJ, 161, 22

Saillenfest, M. 2020, Celest. Mech. Dyn. Astron., 132, 12

Saillenfest, M., Fouchard, M., Tommei, G., et al. 2017, Celest. Mech. Dyn. Astron., 129, 329

Trujillo, C. A., \& Sheppard, S. S. 2014, Nature, 507, 471

van der Walt, S., Colbert, S. C., \& Varoquaux, G. 2011, Comput. Sci. Eng., 13, 22

von Zeipel, H. 1910, Astron. Nachr., 183, 345

Wan, X.-S., Huang, T.-Y., \& Innanen, K. A. 2001, AJ, 121, 1155 


\section{Appendix A: Mutual nodal distance: formulae}

Our statistical analyses are based on studying the distribution of nodal distances between two Keplerian trajectories (one ETNO and one hypothetical planet) with a common focus; therefore, the core of our approach is purely geometrical and gravitational interactions are not directly taken into account. The mutual nodal distance between the orbits of a small body (an ETNO in our case) and an arbitrary planet can be written as (see Eqs. (16) and (17) in Saillenfest et al. 2017):

$\Delta \mathrm{d}_{\mathrm{pn}}=\frac{a\left(1-e^{2}\right)}{1 \pm e \cos \varpi}-\frac{a_{\mathrm{p}}\left(1-e_{\mathrm{p}}^{2}\right)}{1 \pm e_{\mathrm{p}} \cos \varpi_{\mathrm{p}}}$

where

$\cos \varpi=\frac{\cos \omega\left(\sin i \cos i_{\mathrm{p}}-\cos i \sin i_{\mathrm{p}} \cos \Delta \Omega_{\mathrm{p}}\right)+\sin \omega \sin i_{\mathrm{p}} \sin \Delta \Omega_{\mathrm{p}}}{\sqrt{1-\left(\cos i \cos i_{\mathrm{p}}+\sin i \sin i_{\mathrm{p}} \cos \Delta \Omega_{\mathrm{p}}\right)^{2}}}$,

$\cos \varpi_{\mathrm{p}}=\frac{-\cos \omega_{\mathrm{p}}\left(\sin i_{\mathrm{p}} \cos i-\cos i_{\mathrm{p}} \sin i \cos \Delta \Omega_{\mathrm{p}}\right)+\sin \omega_{\mathrm{p}} \sin i \sin \Delta \Omega_{\mathrm{p}}}{\sqrt{1-\left(\cos i \cos i_{\mathrm{p}}+\sin i \sin i_{\mathrm{p}} \cos \Delta \Omega_{\mathrm{p}}\right)^{2}}}$,

$\Delta \Omega_{\mathrm{p}}=\Omega-\Omega_{\mathrm{p}}$, and $a, e, i, \Omega$ and $\omega$ are the orbital elements of the small body, and $a_{\mathrm{p}}, e_{\mathrm{p}}, i_{\mathrm{p}}, \Omega_{\mathrm{p}}$, and $\omega_{\mathrm{p}}$ those of the arbitrary planet. For each random search experiment (each analysis consists of $2 \times 10^{10}$ ), we compute the orbital elements of the putative perturber using the expressions:

$$
\begin{aligned}
& q_{\mathrm{p}}=x+(1000-x) r_{1} \\
& e_{\mathrm{p}}=0.9 r_{2} \\
& a_{\mathrm{p}}=q_{\mathrm{p}} /\left(1-e_{\mathrm{p}}\right) \\
& i_{\mathrm{p}}=80 r_{3} \\
& \Omega_{\mathrm{p}}=360 r_{4} \\
& \omega_{\mathrm{p}}=360 r_{5},
\end{aligned}
$$

where $x=300,400,500$, and $600 \mathrm{AU}$ (see Sect. 2, or $200 \mathrm{AU}$ for Appendix E), and $r_{j}$ with $j=1,5$, are random numbers in the interval $(0,1)$ with a uniform distribution.

\section{Appendix B: Extreme trans-Neptunian objects: Data}

The barycentric orbit determinations used as input data in the uniform Monte Carlo random search discussed in Sect. 3 are shown in Table B.1. The data are referred to epoch 2459000.5 Barycentric Dynamical Time (TDB) and they have been retrieved (as of 11-January-2021) from JPL's SBDB and HORIZONS using tools provided by the Python package Astroquery (Ginsburg et al. 2019).

The orbits of the synthetic ETNOs are obtained using the

\begin{tabular}{|c|c|c|c|c|c|c|c|c|c|c|}
\hline Object & $\begin{array}{c}a_{\mathrm{b}} \\
(\mathrm{AU}) \\
\end{array}$ & $\begin{array}{c}\sigma_{a} \\
(\mathrm{AU}) \\
\end{array}$ & $e_{\mathrm{b}}$ & $\sigma_{e}$ & $\begin{array}{c}i_{\mathrm{b}} \\
\left({ }^{\circ}\right) \\
\end{array}$ & $\begin{array}{l}\sigma_{i} \\
\left({ }^{\circ}\right) \\
\end{array}$ & $\begin{array}{l}\Omega_{\mathrm{b}} \\
\left({ }^{\circ}\right) \\
\end{array}$ & $\begin{array}{l}\sigma_{\Omega} \\
\left(^{\circ}\right)\end{array}$ & $\begin{array}{l}\omega_{\mathrm{b}} \\
\left({ }^{\circ}\right) \\
\end{array}$ & $\begin{array}{l}\sigma_{\omega} \\
\left({ }^{\circ}\right) \\
\end{array}$ \\
\hline $82158\left(2001 \mathrm{FP}_{185}\right)$ & 215.548928 & 0.040005 & 0.841097 & $2.7618 \times 10^{-5}$ & 30.800299 & $3.259 \times 10^{-5}$ & 179.358499 & $4.3407 \times 10^{-5}$ & 6.874579 & 0.00045074 \\
\hline 90377 Sedna & 506.424770 & 0.18758 & 0.849551 & $6.2014 \times 10^{-5}$ & 11.928524 & $3.7219 \times 10^{-6}$ & 144.401511 & 0.00055761 & 311.285511 & 0.0035418 \\
\hline $148209\left(2000 \mathrm{CR}_{105}\right)$ & 221.976439 & 0.6072 & 0.801226 & 0.00056946 & 22.755910 & 0.00058664 & 128.285827 & 0.00029583 & 316.690118 & 0.011843 \\
\hline $445473\left(2010 \mathrm{VZ}_{98}\right)$ & 153.432684 & 0.011897 & 0.776116 & $1.7043 \times 10^{-5}$ & 4.510568 & $9.5639 \times 10^{-6}$ & 117.394668 & 0.00038454 & 313.728058 & 0.00069884 \\
\hline $474640\left(2004 \mathrm{VN}_{112}\right)$ & 327.713834 & 1.5617 & 0.855596 & 0.00067208 & 25.547962 & 0.00027987 & 66.022244 & 0.00043186 & 326.987949 & 0.00928245 \\
\hline $496315\left(2013 \mathrm{GP}_{136}\right)$ & 150.177337 & 0.18997 & 0.726731 & 0.00037413 & 33.538905 & 0.00064333 & 210.727262 & 0.00010633 & 42.569086 & 0.03727751 \\
\hline $505478\left(2013 \mathrm{UT}_{15}\right)$ & 200.156721 & 0.80063 & 0.780530 & 0.0010167 & 10.652044 & 0.0010304 & 191.954169 & 0.00038493 & 252.123877 & 0.032992 \\
\hline $506479\left(2003 \mathrm{HB}_{57}\right)$ & 159.594669 & 0.36652 & 0.761283 & 0.00050502 & 15.500384 & 0.00027768 & 197.871006 & 0.00037845 & 10.837414 & 0.008816 \\
\hline $508338\left(2015 \mathrm{SO}_{20}\right)$ & 164.785707 & 0.020652 & 0.798712 & $2.404 \times 10^{-5}$ & 23.410688 & $3.432 \times 10^{-5}$ & 33.634139 & $1.895 \times 10^{-5}$ & 354.827026 & 0.00055221 \\
\hline $523622\left(2007 \mathrm{TG}_{422}\right)$ & 502.465990 & 0.2381 & 0.929226 & $3.4842 \times 10^{-5}$ & 18.595364 & $3.1757 \times 10^{-5}$ & 112.910531 & 0.00012321 & 285.664088 & 0.00090846 \\
\hline $527603\left(2007 \mathrm{VJ}_{305}\right)$ & 192.002016 & 0.047887 & 0.816750 & $4.288 \times 10^{-5}$ & 11.983654 & $6.5092 \times 10^{-5}$ & 24.382506 & $3.1038 \times 10^{-5}$ & 338.356121 & 0.00089984 \\
\hline 541132 Leleakuhonua & 1077.120640 & 111.53 & 0.939677 & 0.0063597 & 11.671280 & 0.00063416 & 300.993671 & 0.0071997 & 117.944434 & 0.3158 \\
\hline $2002 \mathrm{~GB}_{32}$ & 206.586429 & 0.51121 & 0.828932 & 0.00038669 & 14.192110 & 0.00015226 & 177.043472 & 0.00033413 & 37.047917 & 0.0046496 \\
\hline $2003 \mathrm{SS}_{422}$ & 203.255204 & 148.31 & 0.806561 & 0.16161 & 16.781709 & 0.14714 & 151.041519 & 0.17403 & 211.597889 & 43.173 \\
\hline $2005 \mathrm{RH}_{52}$ & 153.565757 & 0.21296 & 0.746072 & 0.00032216 & 20.445652 & 0.00036748 & 306.109904 & 0.00088931 & 32.512598 & 0.00767 \\
\hline $2010 \mathrm{~GB}_{174}$ & 351.380200 & 22.505 & 0.861811 & 0.010215 & 21.562661 & 0.0051779 & 130.715273 & 0.019788 & 347.236662 & 0.36426 \\
\hline $2012 \mathrm{VP}_{113}$ & 262.065255 & 1.4232 & 0.692739 & 0.0019759 & 24.052063 & 0.0023167 & 90.802707 & 0.0056 & 293.924997 & 0.37222 \\
\hline $2013 \mathrm{FS}_{28}$ & 191.761394 & 98.598 & 0.821344 & 0.0969 & 13.068232 & 0.02486 & 204.638126 & 0.016117 & 102.176514 & 2.4905 \\
\hline $2013 \mathrm{FT}_{28}$ & 294.523622 & 10.063 & 0.852396 & 0.005059 & 17.375255 & 0.0034261 & 217.722701 & 0.0048316 & 40.696900 & 0.16454 \\
\hline $2013 \mathrm{RF}_{98}$ & 363.869961 & 13.352 & 0.900799 & 0.0036143 & 29.538492 & 0.0033747 & 67.635472 & 0.0053263 & 311.757065 & 0.66841 \\
\hline $2013 \mathrm{RA}_{109}$ & 462.902530 & 2.2525 & 0.900602 & 0.00046151 & 12.399719 & 0.00011973 & 104.798695 & 0.005524 & 262.917813 & 0.15134 \\
\hline $2013 \mathrm{SY}_{99}$ & 729.233540 & 24.996 & 0.931474 & 0.0024376 & 4.225431 & 0.0011969 & 29.509257 & 0.0052081 & 32.141187 & 0.11464 \\
\hline $2013 \mathrm{SL}_{102}$ & 314.359140 & 0.70133 & 0.878709 & 0.00025008 & 6.504915 & $7.5898 \times 10^{-5}$ & 94.730847 & 0.0056732 & 265.496106 & 0.055127 \\
\hline $2013 \mathrm{UH}_{15}$ & 173.746767 & 8.3586 & 0.798455 & 0.011307 & 26.080589 & 0.0057534 & 176.542268 & 0.0071993 & 282.865545 & 0.27819 \\
\hline $2014 \mathrm{FE}_{72}$ & 1548.667877 & 440.14 & 0.976632 & 0.0087352 & 20.632449 & 0.0028763 & 336.829059 & 0.0051919 & 133.959137 & 0.064528 \\
\hline $2014 \mathrm{SR}_{349}$ & 298.050876 & 19.924 & 0.840461 & 0.010668 & 17.967979 & 0.0017476 & 34.886583 & 0.014948 & 341.238334 & 0.6312 \\
\hline $2014 \mathrm{WB}_{556}$ & 279.905880 & 2.5421 & 0.847464 & 0.0013182 & 24.157500 & 0.00019211 & 114.891154 & 0.0033985 & 235.333796 & 0.056364 \\
\hline $2015 \mathrm{BP}_{519}$ & 448.721566 & 8.3963 & 0.921446 & 0.0016417 & 54.110682 & $9.9809 \times 10^{-5}$ & 135.213150 & 0.0018968 & 348.058562 & 0.027567 \\
\hline $2015 \mathrm{GT}_{50}$ & 311.140075 & 2.6471 & 0.876545 & 0.0010976 & 8.794999 & 0.0012014 & 46.064057 & 0.0028595 & 128.986870 & 0.11107 \\
\hline $2015 \mathrm{KG}_{163}$ & 680.351485 & 5.8808 & 0.940483 & 0.00037323 & 13.994347 & 0.0011581 & 219.103229 & 0.0017119 & 32.110680 & 0.098552 \\
\hline $2015 \mathrm{KH}_{163}$ & 152.922061 & 0.59238 & 0.738806 & 0.0011085 & 27.137445 & 0.0013782 & 67.572913 & 0.00060792 & 230.813811 & 0.048894 \\
\hline $2015 \mathrm{RX}_{245}$ & 423.304136 & 4.7739 & 0.892386 & 0.0013068 & 12.138092 & 0.0019317 & 8.605207 & 0.0001895 & 65.120605 & 0.045144 \\
\hline $2015 \mathrm{RY}_{245}$ & 225.301756 & 4.5912 & 0.861023 & 0.0028285 & 6.030530 & 0.0010408 & 341.532143 & 0.005986 & 354.533793 & 0.24642 \\
\hline $2016 \mathrm{GA}_{277}$ & 154.842684 & 6.8842 & 0.767982 & 0.011305 & 19.421077 & 0.00034091 & 112.841139 & 0.019248 & 178.521618 & 0.11573 \\
\hline $2016 \mathrm{QU}_{89}$ & 171.465345 & 0.33356 & 0.794425 & 0.00040081 & 16.975710 & 0.00046385 & 102.898107 & 0.0035642 & 303.349937 & 0.082868 \\
\hline $2016 \mathrm{QV}_{89}$ & 171.616289 & 0.22913 & 0.767183 & 0.00036545 & 21.387605 & 0.00041616 & 173.215227 & 0.0011962 & 281.086675 & 0.019706 \\
\hline $2016 \mathrm{SG}_{58}$ & 232.948888 & 0.40228 & 0.849292 & 0.00027193 & 13.220892 & $4.9315 \times 10^{-5}$ & 118.978173 & 0.0023883 & 296.291522 & 0.035779 \\
\hline $2016 \mathrm{TP}_{120}$ & 174.578585 & 24.948 & 0.771693 & 0.038036 & 32.639476 & 0.0005895 & 126.728289 & 0.027144 & 350.990565 & 0.39834 \\
\hline $2018 \mathrm{VM}_{35}$ & 261.464338 & 64.008 & 0.827901 & 0.050434 & 8.479536 & 0.0033767 & 192.409778 & 0.058367 & 302.700112 & 2.6758 \\
\hline
\end{tabular}
expressions:

$$
\begin{aligned}
& a_{\mathrm{s}}=a_{\mathrm{b}}+\sigma_{a} R_{1} \\
& e_{\mathrm{s}}=e_{\mathrm{b}}+\sigma_{e} R_{2} \\
& i_{\mathrm{s}}=i_{\mathrm{b}}+\sigma_{i} R_{3} \\
& \Omega_{\mathrm{s}}=\Omega_{\mathrm{b}}+\sigma_{\Omega} R_{4} \\
& \omega_{\mathrm{s}}=\omega_{\mathrm{b}}+\sigma_{\omega} R_{5},
\end{aligned}
$$

where $R_{j}$ with $j=1,5$, are univariate Gaussian random numbers.

Table B.1. Barycentric orbital elements and $1 \sigma$ uncertainties of known ETNOs with robust orbit determinations.

Notes. The orbit determinations have been computed at epoch JD 2459000.5 that corresponds to 00:00:00.000 TDB on 2020 May 31 (J2000.0 ecliptic and equinox). Input data source: JPL's SBDB. 
Appendix C: Heliocentric orbit determinations: results

If we repeat the calculations discussed in Sect. 3 using heliocentric orbit determinations instead of barycentric ones as input data, we obtain the distributions in Fig. C.1 with central values and dispersions summarized in Table C.1.
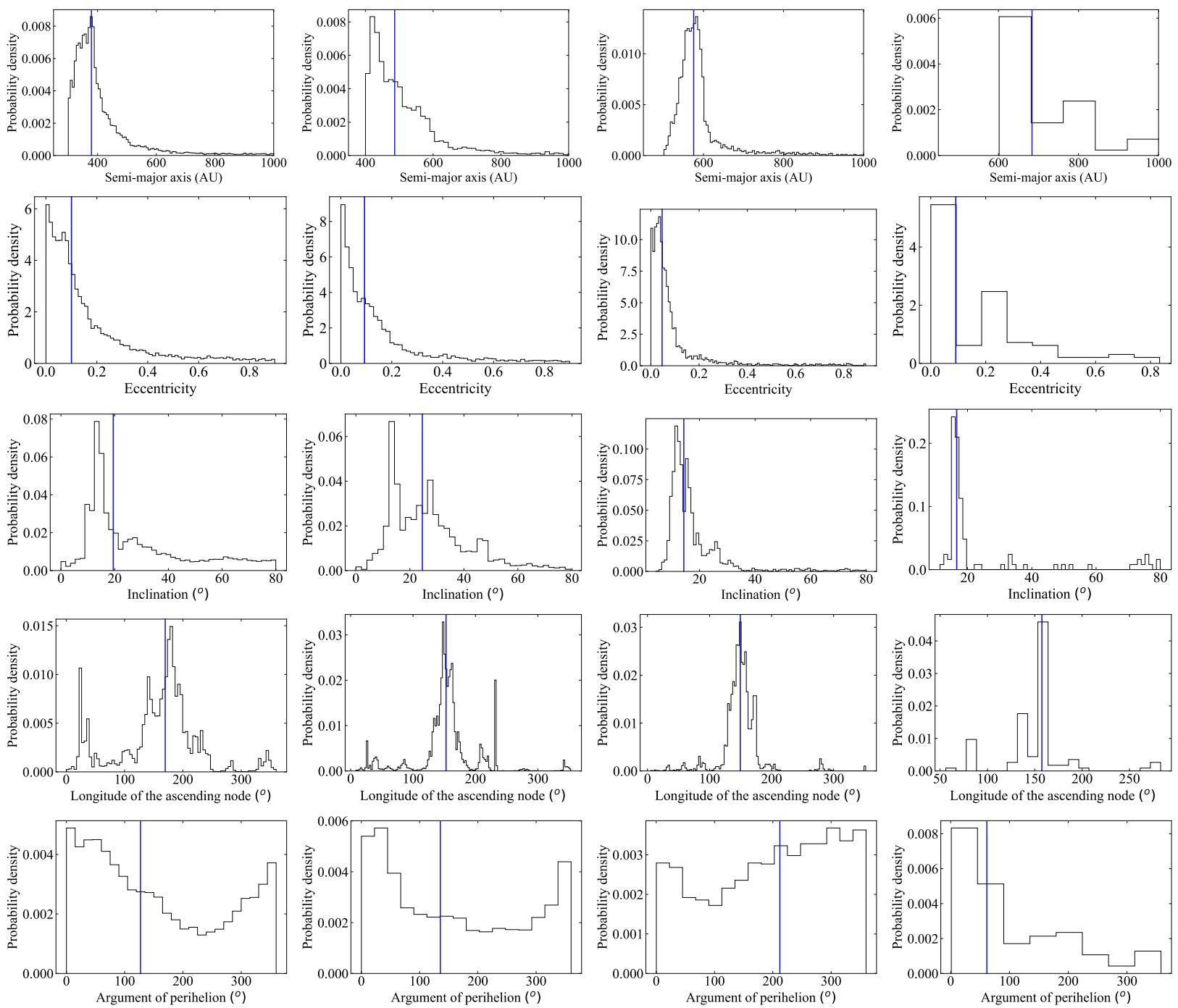

Fig. C.1. Heliocentric orbital elements of putative perturber planets. Distributions of heliocentric orbital elements of planetary orbits that may result in close encounters (under $5 \mathrm{AU}$ for $q_{\mathrm{p}}>300 \mathrm{AU}$, under $7.5 \mathrm{AU}$ for $q_{\mathrm{p}}>400 \mathrm{AU}$, and under $10 \mathrm{AU}$ for $q_{\mathrm{p}}>500 \mathrm{AU}$ and $q_{\mathrm{p}}>600 \mathrm{AU}$ ) with five or more present-day extreme trans-Neptunian objects (ETNOs). Each column of panels shows the cumulative results of $2 \times 10^{10}$ experiments. From left to right: results of imposing $q_{\mathrm{p}}>300 \mathrm{AU}$ (left column, 18369 orbits), $q_{\mathrm{p}}>400 \mathrm{AU}$ (6594 orbits), $q_{\mathrm{p}}>500 \mathrm{AU}$ (3567 orbits), and $q_{\mathrm{p}}>600 \mathrm{AU}$ (right column, 105 orbits). Median values are shown as vertical blue lines. Based on the available data on known ETNOs, the presence of massive perturbers well beyond $600 \mathrm{AU}$ is strongly excluded within the context of the hypotheses centered in this study.

Table C.1. Summary of central values and dispersions of optimal heliocentric orbits.

\begin{tabular}{lcccc}
\hline \hline Orbital parameter & $q_{\mathrm{p}}>300 \mathrm{AU}$ & $q_{\mathrm{p}}>400 \mathrm{AU}$ & $q_{\mathrm{p}}>500 \mathrm{AU}$ & $q_{\mathrm{p}}>600 \mathrm{AU}$ \\
\hline Semi-major axis, $a_{\mathrm{p}}(\mathrm{AU})$ & $379_{-47}^{+112}(378)$ & $486_{-62}^{+129}(420)$ & $576_{-29}^{+45}(584)$ & $684_{-65}^{+299}(645)$ \\
Eccentricity, $e_{\mathrm{p}}$ & $0.10_{-0.07}^{+0.21}(0.01)$ & $0.09_{-0.07}^{+0.18}(0.01)$ & $0.05_{-0.03}^{+0.08}(0.04)$ & $0.09_{-0.08}^{+0.25}(0.05)$ \\
Inclination, $i_{\mathrm{p}}\left({ }^{\circ}\right)$ & $19_{-7}^{+32}(13)$ & $25_{-11}^{+17}(13)$ & $14_{-3}^{+10}(12)$ & $17_{-1}^{+17}(16)$ \\
Longitude of the ascending node, $\Omega_{\mathrm{p}}\left({ }^{\circ}\right)$ & $170_{-77}^{+37}(181)$ & $153_{-19}^{+24}(148)$ & $150_{-16}^{+19}(151)$ & $157_{-26}^{+5}(160)$ \\
Argument of perihelion, $\omega_{\mathrm{p}}\left({ }^{\circ}\right)$ & $127_{-92}^{+180}(7)$ & $136_{-107}^{+179}(35)$ & $212_{-148}^{+102}(305)$ & $62_{-33}^{+144}(22)$ \\
\hline
\end{tabular}

Notes. Median values and 16th and 84th percentiles (absolute maximum in parentheses) from the Monte Carlo random searches whose distributions are shown in Fig. C.1. 
C. de la Fuente Marcos and R. de la Fuente Marcos: Close encounters in extreme trans-Neptunian space

\section{Appendix D: Statistical significance}

In order to verify that our results do not come from statistical artifacts, we randomly scramble the orbit parameters data used as input and repeat the uniform Monte Carlo random searches discussed in Sect. 3 and Appendix C. In these experiments, the set of synthetic ETNOs is such that, for example, the first object may have the value of $a$ of object \#5, $e$ of \#27, $i$ of \#7, $\Omega$ of
$\# 37$, and $\omega$ of \#13. By randomly rearranging the values of the orbital elements of the ETNOs, we retain the original distributions of the parameters, but destroy any possible correlations existing among them. The results of these statistical significance tests are shown in Figs. D.1 and D.2. The distributions in $i_{\mathrm{p}}$ and $\Omega_{\mathrm{p}}$ are flattened and no statistically significant perturbing orbits are produced.
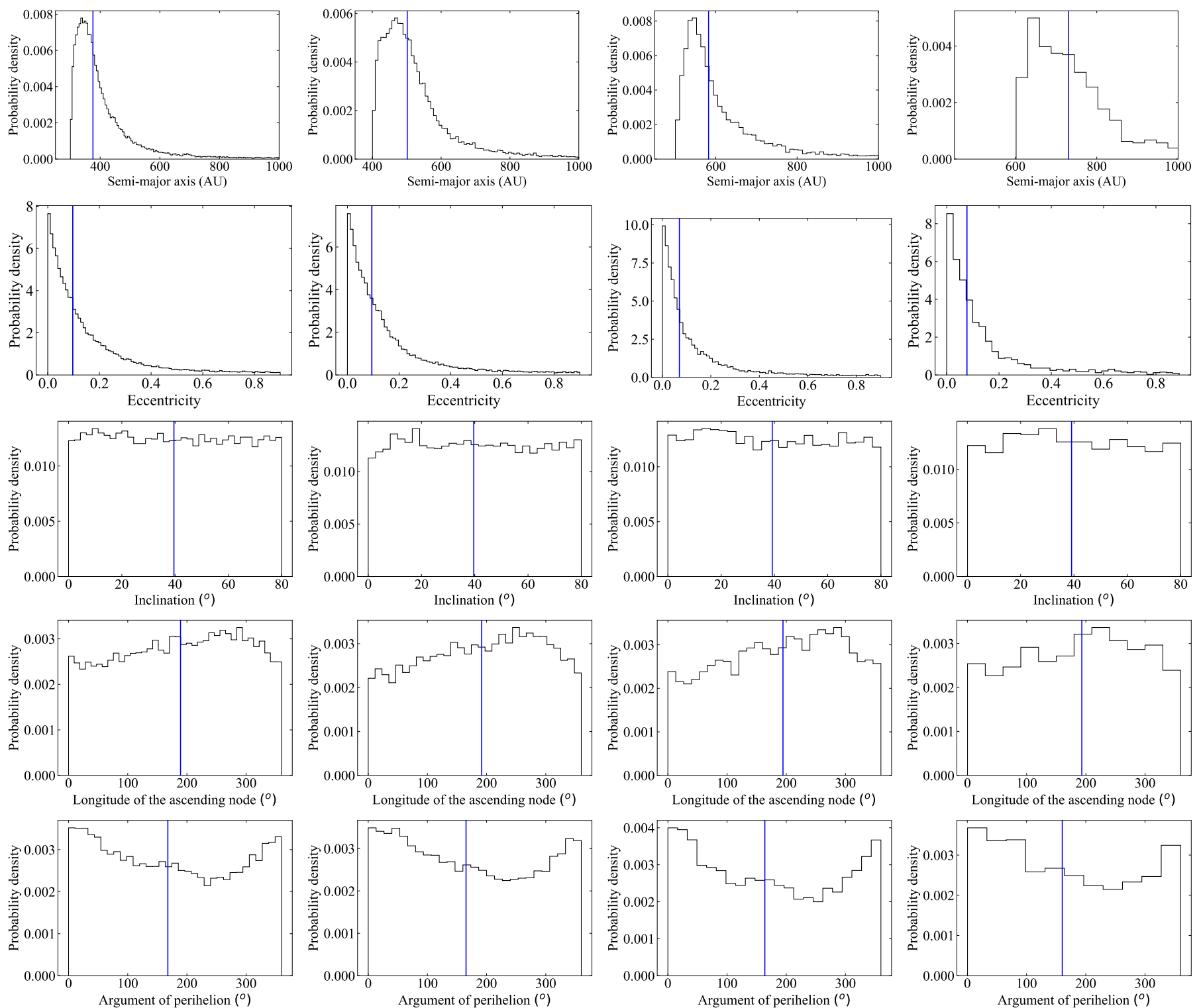

Fig. D.1. Barycentric orbital elements of putative perturber planets. As Fig. 1 but using scrambled data as input. 
A\&A 646, L14 (2021)
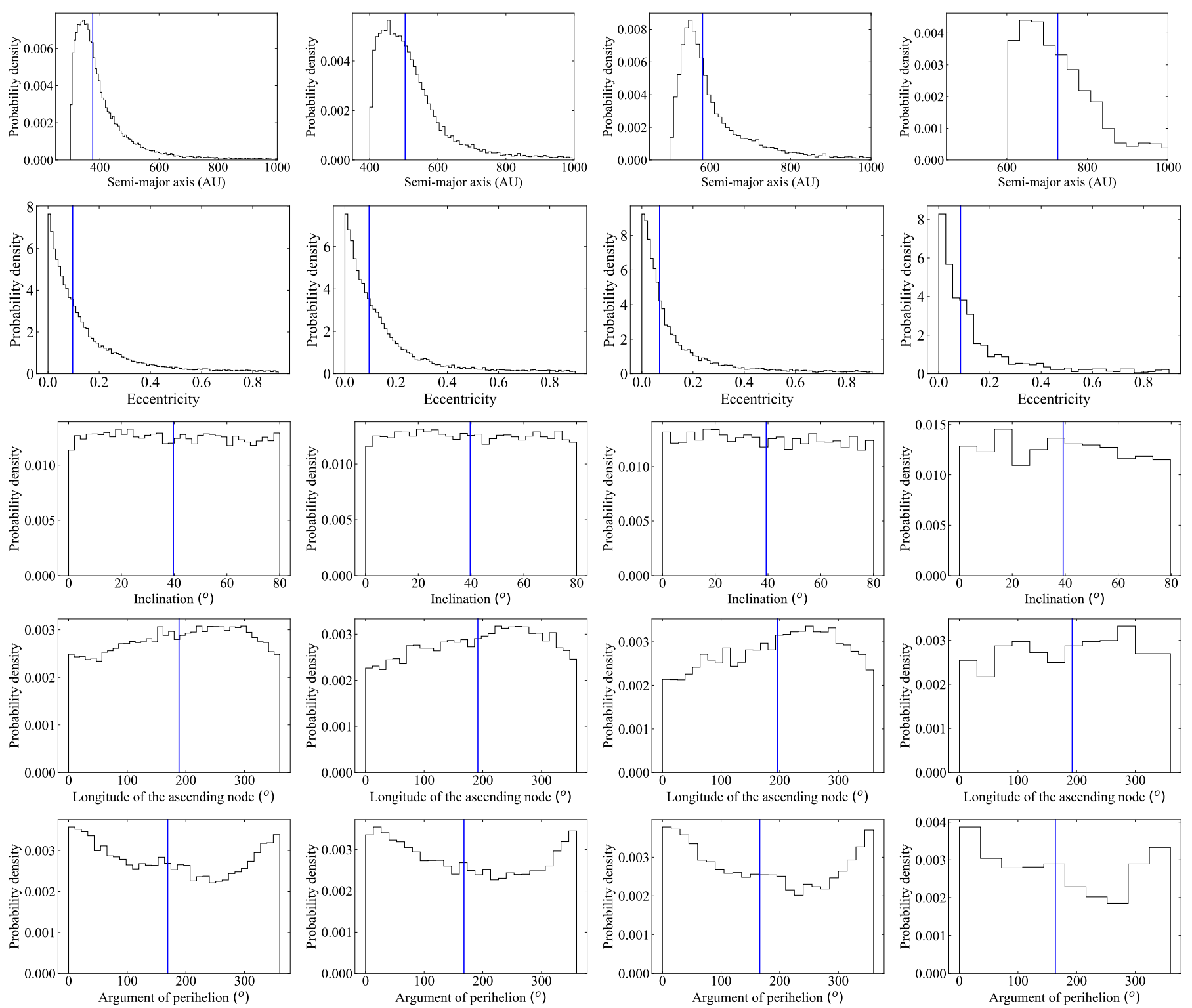

Fig. D.2. Heliocentric orbital elements of putative perturber planets. As Fig. C.1 but using scrambled data as input. 


\section{Appendix E: Results at $200 \mathrm{AU}$}

Fienga et al. (2020) focused on testing for the presence of possible planets at 400, 500, 600, 650, 700, 750, and $800 \mathrm{AU}$ with masses of 5 or $10 M_{\oplus}$. The existence of $5 M_{\oplus}$ planets at 400 or $500 \mathrm{AU}$ is strongly disfavored by their results (see their Fig. 5, top panels). However, a hypothetical Earth-like planet at 200-400 AU from the Sun may still induce significant gravitational effects if close encounters are possible, due to its relatively large value for the Hill radius (e.g., 2.2 AU if $a_{\mathrm{p}}=200 \mathrm{AU}$,
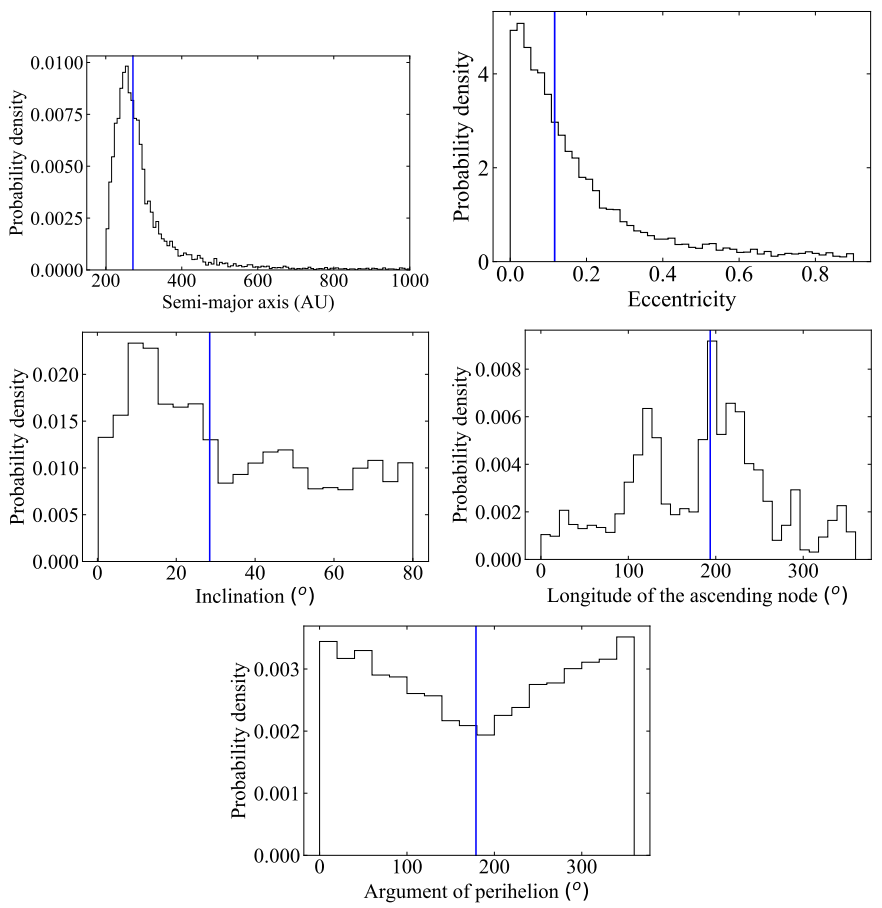

Fig. E.1. Barycentric orbital elements of putative perturber planets. As Fig. 1 but for $q_{\mathrm{p}}>200 \mathrm{AU}$. $e_{\mathrm{p}}=0.1$ and $\left.1 M_{\oplus}\right)$. We repeated the analysis, imposing $q_{\mathrm{p}}>$ $200 \mathrm{AU}$, and we obtained 8234 orbits with a number of potential close approaches in the range between 5-7. Here, we count how many synthetic ETNOs had at least one mutual nodal distance with the planet under $2 \mathrm{AU}$. The median values and 16th and 84th percentiles (absolute maximum in parentheses) from the Monte Carlo random searches whose distributions are shown in Fig. E. 1 are: $a_{\mathrm{p}}=272_{-38}^{+94} \mathrm{AU}(258 \mathrm{AU}), e_{\mathrm{p}}=0.12_{-0.08}^{+0.21}(0.03)$, $i_{\mathrm{p}}=28_{-18^{\circ}}^{\circ+35^{\circ}}\left(10^{\circ}\right), \Omega_{\mathrm{p}}=194_{-90^{\circ}}^{\circ+58^{\circ}}\left(196^{\circ}\right)$, and $\omega_{\mathrm{p}}=179_{-130^{\circ}}^{\circ}$ $\left(350^{\circ}\right)$. 